\title{
Morphological features of papillary muscles in the right ventricle
}

Faculty of Medicine, University "Ovidius" of Constanta

\begin{abstract}
Our study was performed by dissecting formalinized cord, aiming at:the number of bodies of each papillary muscle in the right ventricle, the shape of the muscular body, also the morphological characteristics of the tendon chordaes. The anterior papillary muscles of the right ventricle, were studied on 54 cords, finding a total of 82 papillary muscles. Unique anterior papillary was found in $59.26 \%$ of case, representing $38.10 \%$ of total anterior papillary muscles.

In $29.63 \%$ of cases, the anterior papillary muscle was double and in $11.11 \%$ of cases was threefold. The conical shape we found in $57.32 \%$ of the anterior papillary muscles, and the cylindrical shape encountered in $29.27 \%$ of cases.

In case of double papilaery muscles, sometimes beeing joined at various levels between them, they realized particular aspects in each $2.44 \%$ of cases, presenting the „N" and „H" letter appearance and in $1.22 \%$ of cases one of the two papilary presented an arcuate shape. At the unique papillary in one case $(1.22 \%)$ we have found pyramidal forms and reversed „V" letter.

The valvular tendinous chordae of the anterior papillaries, we have pursued on a number of 78 papillary muscles and we found that most frequently they broke loose from the superior side, in $41.03 \%$ of cases detaching from the tip of conical unique papillaries romthe superior si of those cylindrical. The posterior papillar muscles of the rght ventricle we studied on 42 cords, presenting 69 papillary muscles. Single posterior papillary muscle we found in $52.38 \%$ of cases, in $33.33 \%$ of cases was double, triple
\end{abstract}

Ruxandra Junca (Gheorghitescu)

Faculty of Medicine, University "Ovidius" of Constanta

University Street, No. 1, Campus B

Constanta, Romania

email: rux.gheorghitescu@gmail.com

phone: +40736629594 in $11.90 \%$ of cases and in one case $(2.38 \%)$ the posterior papillry was quadruple. Most commonly, the posterior papillary muscle presents a conical shape, aspect found in $65.22 \%$ of cases, in $27.54 \%$ of cases was cylindric, in $2.90 \%$ of cases we found a papillary muscle in reversed "Y" shape (at double papillary), and in one single case $(1.54 \%)$ we found one arcuated papillary (in one double paillary), one irregular papillary (at unique papillaries) and one papillary in reversed „N" letter (between two muscular bodies of one triple papillary).

Valvular chordaes of the posterior papillary muscles, most frequently broke loose from the tip or superior edge of the papillary, aspect found in $35.48 \%$ of cases. Septal papillary muscles that we studied on 43 cords, were represented either by a number of 3-5 beams, or 5-9 simpe chordae tendons, both tipes could present or not at their origin on the septomarginal strip, a muscular cone. Rarely can present 1-3 large muscular cones that sometimes can be considered true papillary muscles.

We found the arterial muscle cone, present in all cases, beeing represented by chordae bundles arising from a muscular cone and rarely not showing muscular cone at his origin or can be represented by two beams o.f valvular chordae. We encountered one case where septal papillaries were represented by 3 conical muscular bodies, well represented, comparable in size with the anterior and posterior papillary muscles, especially the superior and inferior muscular bodies, the middle beeing shorter. We found in $13.95 \%$ of cases the existence of the posterior's angle papillary muscle of the right ventricle, conical and cylindrical papillary muscle, that sent tendon chordae only to the septal leaflet.

Keywords: right ventricular papillary muscles characteristics. 
ventricle are probably associated with transfer of the papillary muscles from the septum to the back wall of the right ventricle during the phylogenetic evolution.

In the right ventricle, the papillary muscles fluctuate on the number, position and the placement of the tendinous chordae $[1,2,3]$.

The aspects are important in clinical practice, the papillary muscles playing an important role in the contraction of the right ventricle and in the tricuspid valve closure, them preventing the refluation of the ventricular blood in the right atrium. For the surgeont they are important in the $[1,2]$ restorative procedures, papillary muscle dissfunction, mitral valve replacement [1]. Most classic authors and anatomical nomenclature (4), considers taht the papillary muscles, also called pillars $[5,6,7]$ are three: anterior, posterior, septal or medial.

Anterior papillary muscle is the biggest, frequently beeing unique, giving chordae for the anterior valves (multiple) and the posterior $[3,5,7,8,9,10,11]$.

The posterior papillary muscles gives tendon chordae for the posterior and septal valves and can be single, double or triple $[2,3,6,9,12]$, [2,12] finding him quadruple. Medial papillary muscles are represented by tendon chordaes detaching from the septum, either directly or through small muscle cones, genuine miniature papillary muscles [5].

Their chordae insert on septal and anterior valves of the muscle cones, one is constantly, being located at the bottom of the arterial cone (infundibulum), and called by Luschka infundibular papillary muscle or papillary muscle of the arterial cone $[5,7,8,9,11]$.

$[13,14]$ describes outside of the three papillary muscles another two papillary muscles: conal papillary muscle and the papillary muscle of the posterior angle in the right ventricle.

The first is described by Luschka since the 17th century, as the most constant of the septal papillary muscles. Regarding the posterior's angle papillary muscle of the right ventricle, he believes in the fact that they are papillary muscles that cannot be clearly classified as either septal or posterior muscles. The papillary muscles from the posterior angle in the right

\section{Material and method}

Our results on morphological characteristics of the papillary muscles in the right ventricle were obtained from a study conducted on a total of 56 cords, which had 106 muscular bodies in papillary muscles in the left ventricle, of which 58 were for the anterior papillary and 48 for the posterior papillay.

The study was performed on formalinized cords, aiming at: the number of muscular bodies of each papillary muscle, the shape of muscular body, the mode of implanting the base of the muscular body on the ventricular wall, the presence of the parietal chordae and the anastomoses between muscle bodies in case of multiple papillay muscle, the way of ending of valvular distal termination of papillary muscle and the characteristics of the valvular chordae at their origin and at the valvular end.

\section{Results}

The anterior papillary muscles in the right ventricle were studied on 54 cords, finding a total of 82 papillary muscles.

Unique papillary muscle we found in 32 cases (59.26\% of cases), representing $38.10 \%$ of total anterior papillary muscles. In 16 cases $(29.63 \%$ of cases) the anterior papillary was double, the 32 papillary muscles representing $38.10 \%$ of all anterior papillary muscles in the right ventricle.

Tre anterior papillary muscle, formed by 3 muscular bodies, we encountered in 6 cases $(11.11 \%$ of cases), and 18 papillaries representing $21.95 \%$ of 
all anterior papillary muscles.

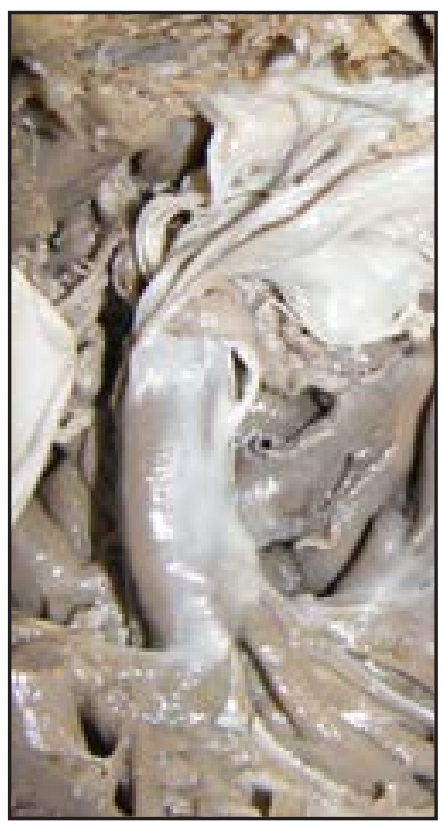

Fig. 1. The right anterior papillary muscle cylindrical base having a branched implant, implanting the ventricular wall being made by two roots of unequal size. Chordae tendon detached from the top end, a tendon stringing himself away from the medial side of body muscle, the middle of its upper half.

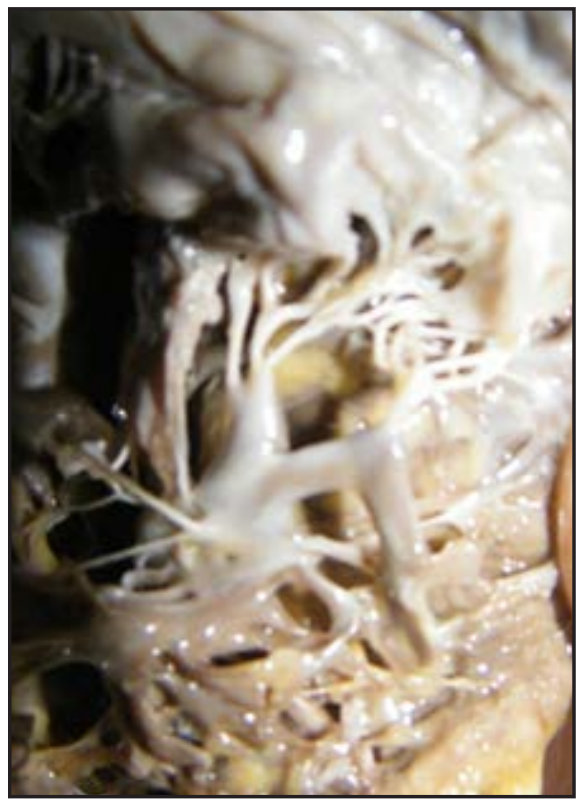

Fig. 2. Double right anterior papillary muscle: muscular body is conical and the other in thereversed " $V$ "letter.
The shape of the anterior papillary muscles varies, finding 8 morphological types. Most frequently the papilllary muscle is conical, finding it in 47 cases $(57.32 \%$ of anterior papillaries), at the unique papillary finding it in 16 cases $(36.36 \%$ of conical papillaries), at double papillary in 22 cases $(50 \%$ of cases) and at triple papillary in 6 cases (13.64 of cases). Cylindrical shape of the anterior papillary, we found in 26 cases $(29.27 \%$ ), and the triple papillary in 5 cases $(20.83 \%)$.

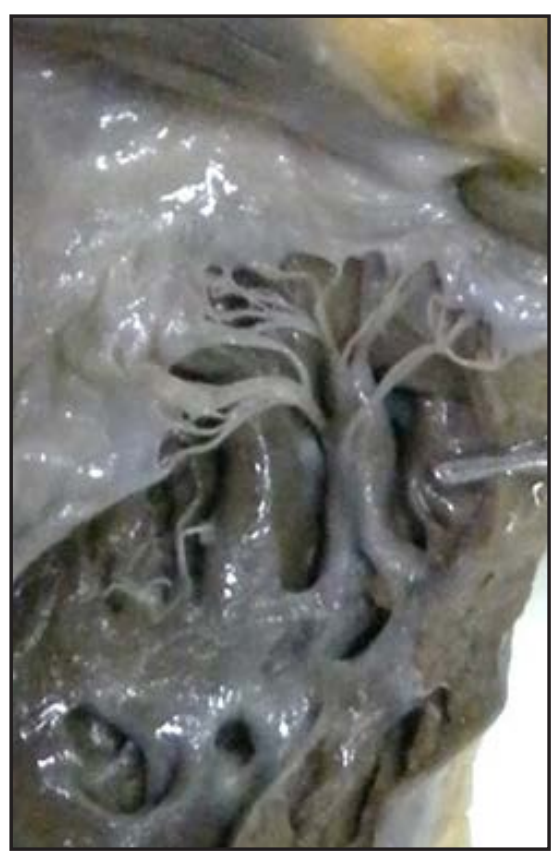

Fig. 3. Double right anterior papillary muscle, muscle consists of two conical bodies, with the bulky side being one root having the second root medially of its basis in the implantation of the ventricular wall. Muscular body side is linked by a bridge to the papillary muscle root medial side, closer to the base, creating the appearance of the letter " $H$ ". Valve chordae to emerge from its peak, showing a lateral parietal stringing above its base. Medial papillary has in the tip, two cones that fall muscle valve chordae. Valve chordae its level above the middle and detach from the anterolateral.

In case of double papillary muscles, in half cases both muscles presented the same shape and also in half of the cases they had different form. Being sometimes joined at various levels by muscular bridges or tendon strings, the double papillary muscles realises particular aspects, in 2 cases $(2.44 \%$ of all 
cases) presenting the „N" letter appearance, reversed „N” letter appearance and also „H” letter appearance.

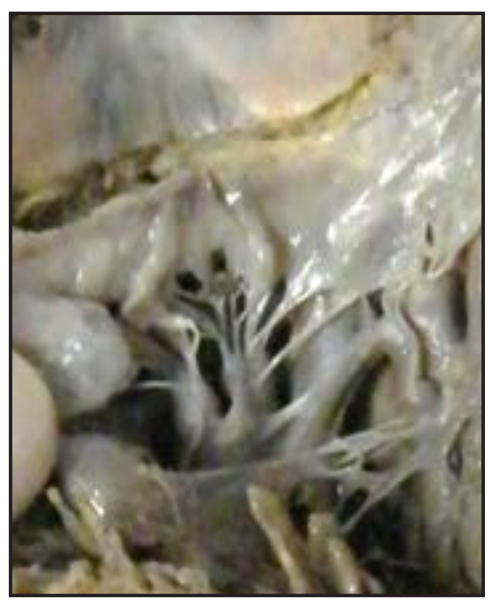

Fig. 4. The right anterior papillary muscle triple, 2 bodies (lateral and middle) is tapered, and the medial arcuate, situated at a distance of medial papillary. The first two bodies in the middle portion of the muscle are united by a bridge oblique muscle superolateral inferior and mediallateral (aspect of letter " $N "$ "), and medial and middle muscular bodies are joined in the middle portion by an oblique tendon stringing supero- medial vertical segment of the body from muscular arched toward the middle.

Also in double papillaries, I encountered one case $(1.22 \%)$ in which one of the two papillary muscles present an arcuate shape. To the unique papillary muscles, in one case $(1.22 \%$ of cases $)$ I have met piramidal shape and reversed "V" letter appearance. In the case of triple papillary muscles, in 4 cases $(66.67 \%$ of the cases) the three muscles showed the same shape (either cylindrical or conical), and in 2 cases (33.33\% of the cases) the muscles were different forms. The most common is the basis of the right anterior papillary muscle unique aspect found in 52 cases $(63.41 \%$ of cases) in the remaining cases showing papillary muscle base or two root implant, in 24 cases $(29.27 \%$ of the) or three roots, in 6 cases (7.32\% of cases). The tendoncordae of the valve in the anterior papillary muscle, we have pursued a number of 78 papillary muscles and found that they most frequently broke loose on top, in 32 cases (41.03\% of cases) and detaching only from unique papillaries tip or the upper face of the cylindrical, in 14 cases (17.95\% of cases), emerges from the top of the papillary in 6 cases $(7.69 \%$ of cases) from the left to the supero-lateral and in 4 cases $(5.13 \%$ of cases) in the anterior upper face. We met outside valve chordae: parietal chordae, who started from different levels of papillary muscle to the ventricular wall; interpapilare chordae, lying between double or triple papillary muscles and interpapilary chordae arranged between the anterior and posterior papillary; and chordae disposed between papillary roots. In one case I found a valvular chordae arrising from an interpapillary muscule bridge.

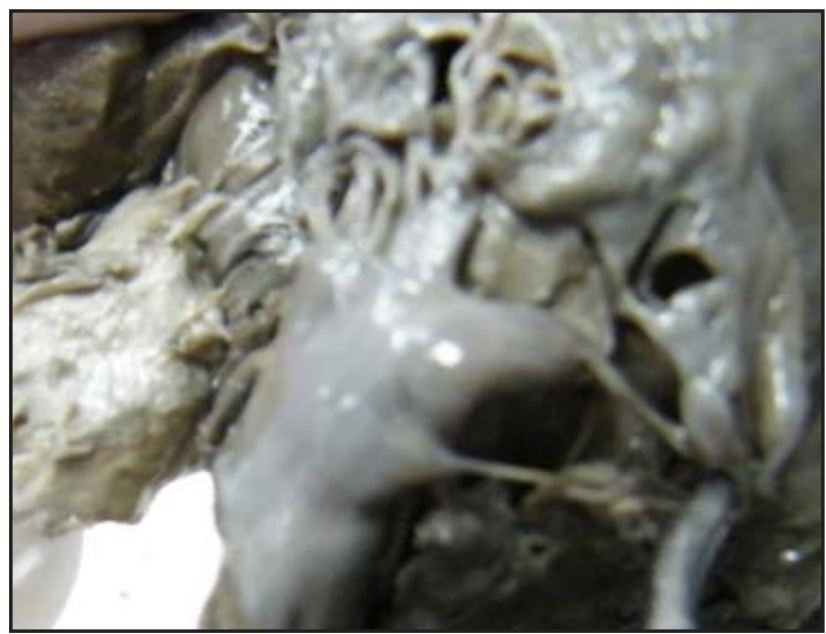

Fig. 5. posterior papillary muscle as cylindrical, based on presenting two unequal roots. At the upper end presents four cones muscle detached from the which the valve chordae. From the middle of the face medial muscle emerges a cone gives a closer stringing valvular branched valve to valve septal.

Posterior papillary muscle of the right ventricle I studied in a total of 42 cords, presenting 69 papillary muscles. Single papillary muscle I have encountered in 22 cases $(52.38 \%$ of cases), 22 papillary muscles representing $31.88 \%$ of the posterior papillary muscles); 14 cases (33.33\% of cases) had posterior papillary muscle doubles, 28 papillary muscles representing $40.58 \%$ of the posterior papillary muscles; in 5 cases $(11.90 \%$ of cases) papillary muscle was triple the 15 muscles representing $21.74 \%$ and in one case $(2.38 \%)$ posterior papillary muscle was quadruple, the 4 papillary muscles representing $580 \%$ of papillaries. The most commonly posterior papillary muscle present a conical aspect found in 
45 cases $(65.22 \%$ of cases). In 16 cases $(35.56 \%$ of cases) conical muscle I met in single papillary in 17 cases $(37.78 \%$ of cases $)$ in double papillary in 10 cases $(22.22 \%$ of cases $)$ in triple papillary and in 3 cases $(6.67 \%$ of cases) in quadruple papillary.

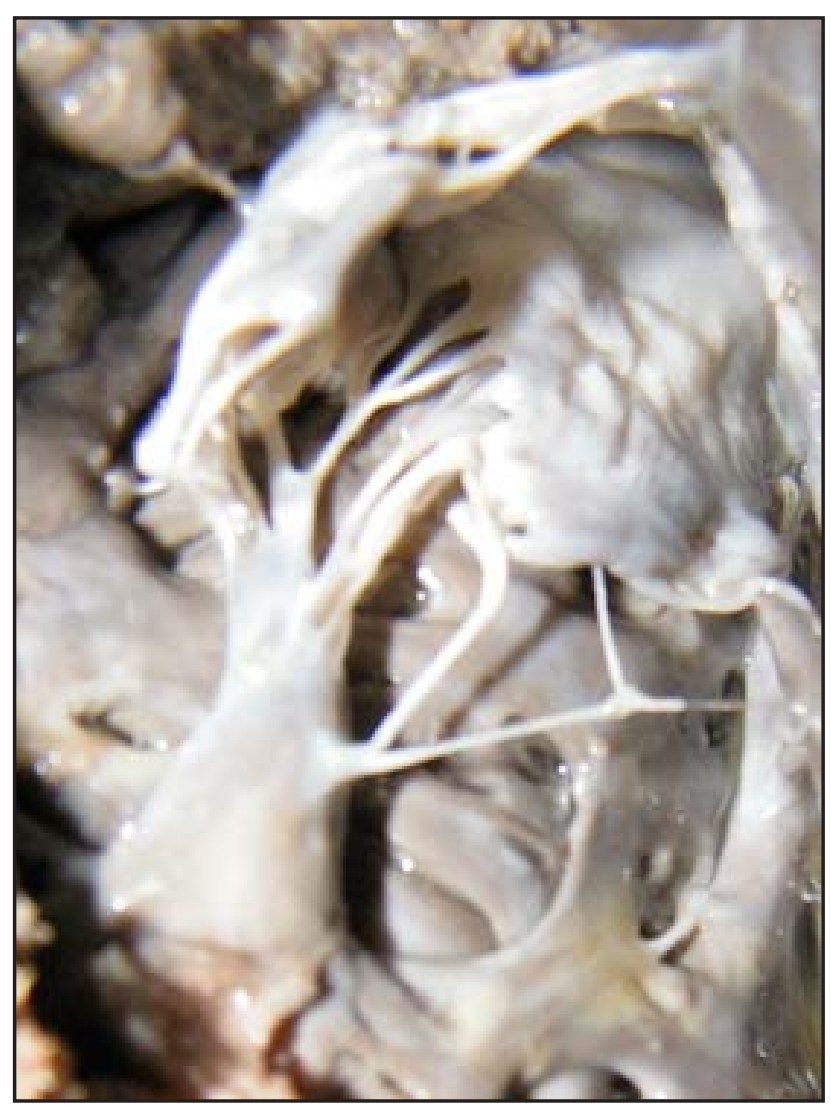

Fig. 6. posterior papillary muscle sole, tapered perforated base. Valve chordae tendon becomes detached from the papillary tip on its side and medial superolateral. From the medial portion of the upper medium emerges a stringing which branches into a ram and a ram valve superior parietal, inferior.

Cylindrical papillary muscle I encountere in 19 cases $(27.54 \%$ of cases) in 5 cases $(26.32 \%$ of cylindrical muscles) muscle cone I met in unique papillary in 8 cases $(42.21 \%$ of cases $)$ in double papillary in 5 cases ( $26.32 \%$ of cases) in triple papillary and in one case (5.26\% of cases) in quadruple papillary . In 2 cases ( $2.90 \%$ of cases) I met a papillary muscle shaped in reversed "Y" letter (to the double papillary) and in one single case ( $1.45 \%$ of cases), I met one arcuate papillary (a double papillary), an irregular papillary (the unique papillary) and papillary in the form of "N" reversed letter (between two bodies of a triple papillary muscle). In cases of double papillary muscles in $50 \%$ of cases they were the same shape and all in $50 \%$ of cases they had a different form. In cases of triple papillary muscle, in 3 cases they had the same shape and in two cases they wer different in their presentation. In the only case with quadruple papillary muscle, 3 bodies were conical muscular body and one musclar body was cylindrical. In 52 cases $(75.3 \%$ of the cases) the posterior papillary muscle of the right ventricle presented single base of implantation, in 15 cases $(21.74 \%$ of the cases $)$ the base of the papillary muscle implantation presented two roots, and in 2 cases $(2.90 \%$ of cases $)$ present three roots.

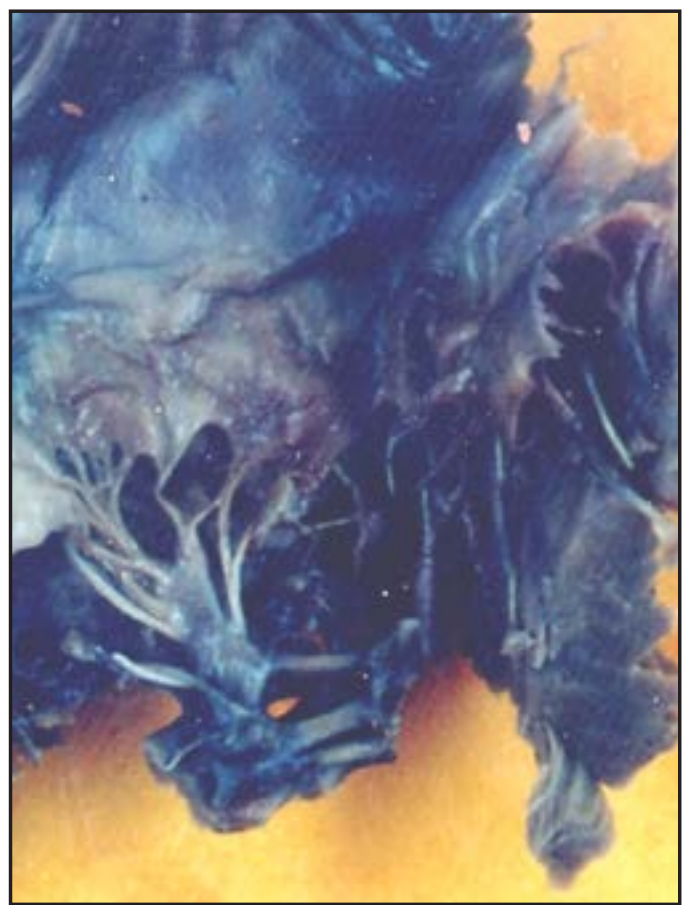

Figure 7. Right posterior papillary muscle, irregular, unique, widened the basis of, the valve chordae emerging from the top, in the middle of his face, side (two) and medial.

Valvular chordaes of the posterior papillary muscle most frequently broke loose from the tip of the conical papillary or from the upper edge of the cylindrical papillary aspect found in 22 cases (35.48\% of cases). In 12 cases ( $19.35 \%$ of cases) the 
chordaes broke loose from the superolateral average in 10 cases $(16.13 \%$ of cases) and also in 10 cases from the superior face (16.13\% of cases) on the front (antero-superior, antero-inferior and antero-medial) and in each 4 cases $(6.45 \%$ of cases) valvular chordae broke loose in the middle of the face or medial side.

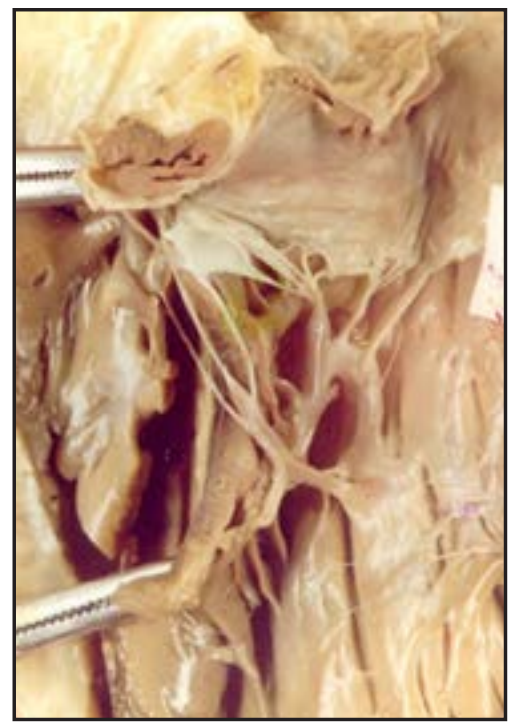

Fig. 8. posterior papillary muscle as double the medial arch, while the side placed higher and lower thinner, "Y" back. Arcuate papillary valve chordae on emerging from the convexity, and the anterior-medial face and the front of the descending portion (side) of the arch (" $U$ " or horseshoe back). From the " $Y$ " valve chordae returned turn one, on top, and on its sides superolateral lateral and medial superolateral.

Septal papillary muscles, which I watched on a number of 43 cords, arrising from septolateral trabeculae, are represented either by a number of 3-5 beams (which will later branch into a variable number of valvular chordaes) or 5-9 simple tendinous chordaes, or both types can present, at their origin the muscle cone, variable in size. Rarely, cam present 1-3 large muscular cones that can be considered true papillary muscles. There are cases when bundles of tendon chordae can be reassigned to their origin, later branching, other septal beams being located away from them.

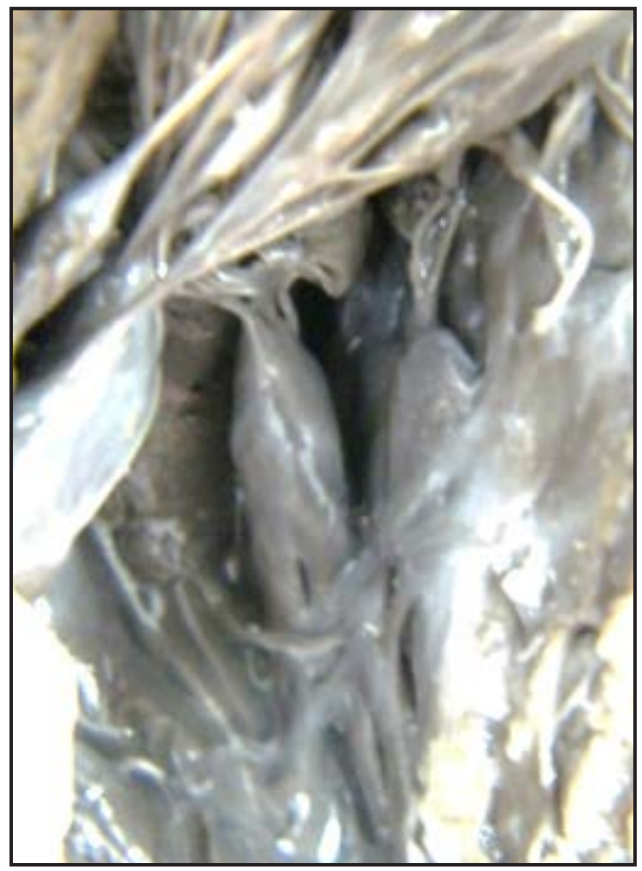

Fig. 9. posterior papillary muscle as triple, three bodies being tapered muscle. The body side is muscular perforated near the middle of his roots and presents the two implantation, the average being drilled. Lateral muscle between the bodies of a bridge there and medium oblique muscle superolateral inferior and medial-lateral (aspect letter " $N$ " returned).

Arterial muscle cone I found present in all cases, being represented by bundles of chordae arising from a muscular cone and rarely not showing muscular cone in its origin. Can be represented by two beams of valvular chordae, located one below to the other, a situation that can give septal valvular chordae and (the lower one), the most common arterial muscle cone giving only anterior leaflet chordae. Chordae beams originating from the strip in arterial muscle cone, can be arranged underneath one another regularly or irregularly located, featuring a zig-zag and unevenly spaced. The beams of the valvular chordae branches at different distances from the origin: closer trabeculaes, midway valvular trabeculaes or valve closer that inserts. Insert the valve chordae can be the free edge of the respective valve (anterior or septal) and ventricular on his face. There are cases where septomarginal trabeculae can present transverse perforations or oblique chordae beams and can sometimes arise at their level or on them. 


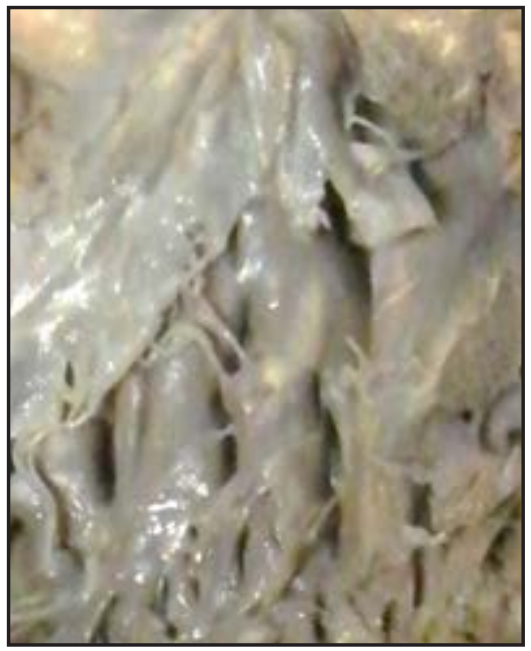

Figure 10. Posterior papillary muscle muscular bodies 4, conical side, lower in height; Middle bifurcated implant above the bottom of which is perforated in a thin cylindrical body side and a medial tapered body having a perforated base and having three unequal root implantation; Papillary third, medial, is cylindrical being bound by a stringing tendon in the middle portion of the body medial papillary middle and above the base between the two bodies muscle connection is achieved through a bridge muscular oblique superolateral inferior and lateral-medial (from middle towards the medial papillary); the medial papillary come off valve chordae of a cone on its side muscle above its middle portion; all of this cone detaches and cordage that link it to papillary middle and lower superolateral oblique medio-lateral.

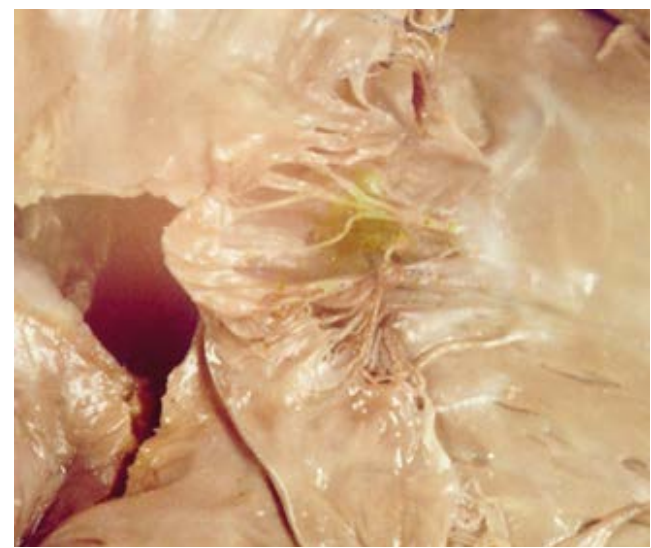

Fig. 11. Muscle is an arterial cone beam chordae, which do not contain muscular in origin. Under it chordae tendon are tiered bundles and chordae free single muscle cone, branched closer to home, dragging on septal valve.

The first beam of sub Conal give muscle valve chordae and earlier.

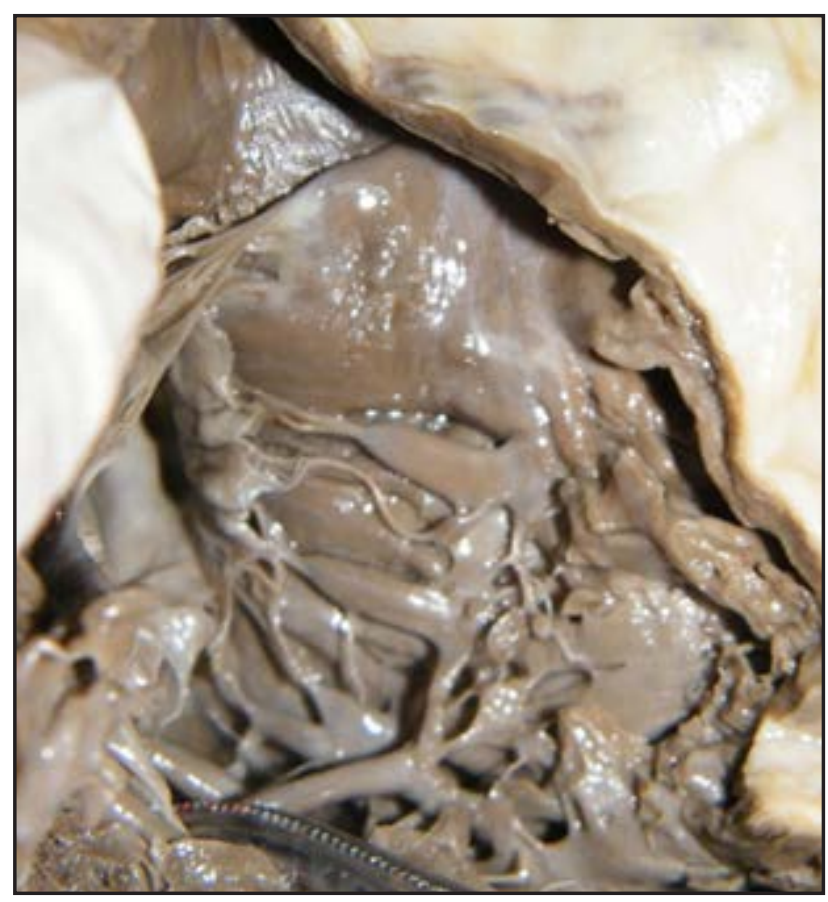

Fig. 12. septal papillary muscles are represented by three conical muscular bodies, comparable in size with the front and rear papillary muscles, especially the upper and lower muscular bodies, the middle being shorter. From their tops off valve chordae one beam for septal valve, which branch off closer valve chordae tendon 3-4. Papillary muscle of arterial cone is represented by two components: a cone that gives muscle and the anterior leaflet chordae tendon without cone beam, the septal branch off the valve. The bottom of the strip septomarginale emerges a papillary muscle angle rear right ventricle, represented by a body muscular cylinder that higher splits into two cones muscle at the top of which is cesprinde one beam chordae giving chordae tendon for septal valve.

I encountered one case where septal papillary muscles were represented by three muscular bodies tapered well represented, comparable in size with the front and rear papillary muscles, especially the upper and lower muscular bodies, the middle being shorter. From their peaks one beam himself valvular chordae for septal valve, which branch closer to valve in 3-4 tendon chordae. In this case, the papillary muscle of arterial cone was represented by two components: a muscular cone and a tendon without cone. From the top of the muscular cone broke lose 5 muscle beams, 
which later will branch out into tendon chordaes (closer to origin) for the anterior valve. Under the cone, the arterial muscle cone beam shows a papillary who send septal valve chordae.

I encountered 6 cases ( $13.95 \%$ of cases) that we considered that the existence of a posterior papillary muscle of the posterior angle in the right ventricle papillary muscle, conical or cylindrical papillary muscle, who had sent septum valve tendon chordae.

\section{Discussions}

Comparing our results with those found in the literature that I have had the opportunity to consult, we found similarities but also differences, sometimes quite conclusive. Anterior papillary muscle of the right ventricle [12] is present in all cases, the aspect that I found in the study. Regarding the number of the anterior papillary muscles (3) mentions that frequently is unique, and [12], a study of 79 cords, finds unique in $81 \%$ of cases, the remaining $19 \%$ of cases being double, not mentioning the possibility of a higher number of anterior papillary muscles. We found anterior papillary unique in a smaller percentage with $21.74 \%$, double papillary found in a higher percentage of $10.63 \%$, meeting, however, cases of triple muscles. For [6] anterior papillary muscle is conical, and not mentioning other form thereof. We have described eight variants of papillary form above, single and multiple variants of conical papillary, cylindrical in "V" and "H" being mentioned by $[14,15]$, but without indicating percent. We do not found versions of "V" but the form of reversed "V" letter, but we described other variants: the "N" in "N" turned and arched. For $[5,7]$ anterior papillary chordae, numbering about 10 , emerging from the cone on top of the papillary muscle, we encounter these valvular chordae can be drawn on the faces of the medial, lateral or anterior papillary muscle, both anterior and posterior.

Posterior papillary muscle, [5] considers to be represented by 1-3 muscular bodies, [6] as consisting of 1-2 muscular bodies. [3] mentions three variants:
1. a single papillary muscle, but bulky, having branched top; 2. a posterior muscle group consisting of 2-3 muscle bodies; 3 . absence of posterior papillary muscle, tendon strings that would be matched by inserting themselves directly or through small dimples on the ventricular walls, septal and posterior. Posterior papillary muscle of the right ventricle [12] finds present in all cases, and we also observed in the study. Comparing our results with those described by [12], we find that we have found the single most common papillary muscle with $26.97 \%$, the double a smaller percentage of $12.97 \%$, the triple lower by $4 \%$ and the quadruple lower $3.92 \%$.

Septal papillary muscle after $[5,6,8]$ is represented by chordae that fall either directly or by means of small fleshy forms, genuine papillary muscles in miniature. After [3] septal papillary muscle can sometimes have 1-2 dimples, suggesting the presence of a papillary muscle. We have found that the most frequently at septum chordae are drawn both by muscle cones, whose number is variable and directly, with no muscle cone, encountering only rare cases where valvular chordae emerge from one morphological type. [12] considers that when valvular chordae emerge without any muscle cone, absent variant that we found in $21.5 \%$ of cases. When there are muscle cones, which may be single $(41.7 \%$ of the cases), double (16.5\% of the cases), triple $(12.7 \%$ of the cases) and quadruple (7.6\% of the cases). [16] on a study of 111 cords, found the presence of papillary muscle of the arterial cone in $82 \%$ of cases, we found him present in all cases. We considered separately lung and papillary muscle cone valvular chordae rest, underlying it. In lung papillary muscle cone, I found it present in $64.29 \%$ of cases muscle, muscular cone not present in $21.43 \%$ of cases, both versions existing in $14.28 \%$ of cases, in which the papillary muscle was formed by 2-3 tendon bundles, whose string tendon insert both on anterior valve and on the septal valve. If underlying septal papillary muscle arterial cone, we found the following situation: the existence of papillary muscle only I met only in one case $(2.38 \%$ of cases); the existence of the chordae without the muscle cone 14 cases (33.33\% of cases); both versions I've encountered most frequently in 27 cases (64.29\% of cases), and these muscle cone 1-3. 


\section{Eonclusion}

It is noted variability of papillary muscle of the right ventricle in the number, shape, chordae tendon that fall from their level and as regards the existence of links between bodies muscle of the papillary muscles between two or three papillary muscles, and links between the papillary muscles and ventricular wall properly. Particular importance is to study papillary muscle morphology, especially for practitioners, especially cardiac surgeons. [10] states that a heart attack can also achieve papillary muscle myocardium. A decrease in blood perfusion can lead to muscle tissue necrosis and papillary muscle rupture, in which the valve does not close properly and blood will reflow toward the atrium during ventricular systole. By [11], papillary muscles start to contract previously ventricular contraction. They lie so close cusps and chordae tendon. Their contraction lasts throughout the ventricular systole, so blocking blood to reflux into the atrium. For $[13,14]$ study of papillary muscle tendon strings distribution is particularly important in cardiac surgery techniques, progress in the repair of the tricuspid valve replacement, after traumatic regurgitation, surgical repair of tricuspid valve and invasive procedures valvuloplasty.

\section{References}

1. Hosapatna, M., Souza, D.A., Das, A., Padamashali, S., Ankolekar, V \& Souza A.D.S. (1995). Morphology of human papillary muscles in adults: a cadaveric study. J Heart Valve Dis. 4 (suppl 2), 115-23

2. Xanthos, Th., Dalivigkas, I. \& Ekmektzoglou, K. (2011). Anatomical variations of the heart valves and papillary muscles of the right heart. Italian J. Anat.Embr. 116 (2), 111-126

3. Filipoiu, Fl.M. (2012). The cord. Anatomy, landmarks embriologice and myocardial notions infrastructure. (pp. 148-150; 167-169). Buchares: Ed. Prior.

4. ******** (1998). Terminology Anatomica. International Anatomical Terminology. Anatomical Terminology federal Committee on. (pp. 75-77). Stuttgart: Ed. Thieme

5. Test, L. (1921) Cœur. In Traité d'anatomie humaine. Angéiologie, Livre IV, Ed. Gaston Doin, Paris. (pp. 3-59).

6. Rouvière H. \& Delmas, A. (1997). Anatomy du cour. In: Anatomie Humaine. Descriptive topographique et fonctionnelle. Tome 2. Tronc. Ed. Masson, Paris, (pp. 125-157).

7. Bouchet, A. (1991) Cuilleret Anatomy topographique J., et fonctionnelle descriptive. 2. Le cou, Le thorax. Ed. Simep, Paris, pp. 985-993

8. Standring, S. (2005). Gray's Anatomy. The Anatomical Basis of Clinical Practice. (pp 1003- 1004). Edinburgh : Ed. Elsevier Churchill Livingstone,

9. Christides, C. (1994). Cabrol et du cour du Anatomy C. pericarditis. In: Le Chevrel thump, Anat. Clin., (pp 141-172). Paris: Ed. SpringerVerlag.

10. Schunk, M., Schulte, E. \& Schumacher, U. (2005). Atlas d'anatomy. Cou et organes internes. (pp. 104-106). Paris:Ed Maloine.

11. Moore, K. \& Dalley, A. (2001). Anatomy doctor. Aspects fondamentaux et Cliniques applications. (pp. 127-131) Brussels:Ed. De Boeck Université.

12. Nigri, G.R., Di Dio, L.J.A. \& Baptista, C.A.C. (2001). Papillary muscles and tendinous cords of the right ventricle of the human heart morphological characteristics. Surg Radiol Anat . 23 (1), 45-49

13. Skwarek, M., Hreczecha, J., Grzybiak, M. \& Kosiński, A. (2005). Remarks on the Morphology of the papillary muscles of the right ventricle. MorphoL foil. 64 (3), 176-182.

14. Skwarek, M., Hreczecha, J., Dudziak, M., Jerzemowski, J. \& Grzybiak, M. (2005). The Morphology and distribution of the tendinous chords and Their relation to the tricuspid valve papillary muscles in the heart of the human. Morphol film. 2005, 66 (4), 314-322

15. Język, D., Jerzemowski, J. \& Grzybiak, M. (2003). Provision of tricuspid valve leaflets by 
septal papillary muscles in the right ventricle of human and other mammal hearts. Morphol film.

(Warsz). 62 (3), 309-311. 